\title{
El género Pyxine (Lecanorales, Physciaceae) en la Reserva de la Biosfera Yaboty (Misiones, Argentina)
}

\author{
The genus Pyxine (Lecanorales, Physciaceae) in the Yaboty \\ Biosphere Reserve (Misiones, Argentina)
}

Instituto de Botánica del Nordeste (IBONE-UNNE-CONICET), Sargento Cabral 2131, CC 209, CP 3400, Corrientes, Argentina.

*mariainessarlej@ratti.net

Citar este artículo SARLEJ, M. I. 2019. El género Pyxine (Lecanorales, Physciaceae) en la Reserva de la Biosfera Yaboty (Misiones Argentina). Bol. Soc. Argent. Bot. 54: 161-168.

DOI: http://dx.doi. org/10.31055/1851.2372.v54. n2.24361

Recibido: 4 Septiembre 2018 Aceptado: 5 Febrero 2019 Publicado: 30 Junio 2019 Editora: María Victoria Vignale

\author{
María Inés Sarlej* ${ }^{*}$
}

\section{SUMMARY}

Background and aims: Pyxine (Lecanorales, Physciaceae) is an interesting genus, which is little known in the Argentinian territory, for this reason a study of the species presents in the Yaboty Biosphere Reserve (Misiones, Argentina) is proposed.

M\&M: The identification of the species was carried out following the methodology conventionally used for the study of lichens, which includes a morphological and chemical analysis. The macroscopic characters were studied with a stereoscopic microscope. In order to determine the type of plectenchyma and study the morphology of ascospores, freehand sections were made, which were subsequently mounted in $5 \%$ potassium hydroxide and observed under an optical microscope. The identification of the lichen substances present was carried out by means of specific color reactions with $10 \%$ potassium hydroxide, ultraviolet light chamber test and thin layer chromatography with solvent $A$.

Results \& Conclusions: In this contribution Pyxine daedalea, Pyxine katendei, Pyxine maculata and Pyxine sorediata are cited for the first time for Argentina. It is presented a dichotomous key, a brief description of the species in the area, geographical distribution, observations and illustrations of each of them. It is considered that this work is a great contribution for further taxonomic and ecological investigations.

\section{KeY WORDS}

Conservation area, lichens, Pyxine, taxonomy.

\section{RESUMEN}

Introducción y objetivos: Pyxine (Lecanorales, Physciaceae), es un género interesante, poco conocido en el territorio argentino, por ello se presenta un estudio de las especies, presentes en la Reserva de la Biosfera Yaboty (Misiones, Argentina).

M\&M: La identificación de las especies fue realizada siguiendo la metodología convencionalmente utilizada para el estudio de los líquenes, que incluye un análisis morfológico y químico. Los caracteres macroscópicos fueron estudiados con un microscopio estereoscópico. Para determinar el tipo de plecténquima y estudiar la morfología de las ascosporas se realizaron cortes a mano alzada, que fueron posteriormente montados en hidróxido de potasio al $5 \%$ para ser observados en microscopio óptico. La identificación de las sustancias liquénicas presentes fue realizada mediante reacciones puntuales de color con hidróxido de potasio al $10 \%$, test en cámara de luz ultravioleta y cromatografía de capa delgada con solvente A.

Resultados \& conclusiones: En esta contribución se citan por primera vez para la Argentina: Pyxine daedalea, Pyxine katendei, Pyxine maculata y Pyxine sorediata. Se presenta una clave dicotómica y una breve descripción para las especies en el área, distribución geográfica, observaciones e ilustración de cada una de ellas. Se considera que este trabajo es un gran aporte para posteriores trabajos taxonómicos y ecológicos.

Palabras Clave

Área de conservación, líquenes, Pyxine, taxonomía. 


\section{INTRODUCCIÓN}

El género Pyxine Fries, se caracteriza por presentar un talo folioso, blancuzco, grisáceo a verdoso generalmente con pruina abundante $\mathrm{y}$ persistente, frecuentemente maculado $\mathrm{y} / \mathrm{o}$ pseudocifelado, lóbulos discretos a imbricados, corteza superior paraplectenquimática con lichenoxantona o atranorina, corteza inferior prosoplectenquimática, superficie inferior marrón oscura a negra con ricinas concoloras, médula blanca o pigmentada, apotecios lecanorinos a zeorinos frecuentemente lecideinos y ascósporas de tipo Dirinaria (Tuck.) (Scutari, 1995; Jungbluth, 2010).

Pyxine, es un género cosmopolita, poco estudiado en Argentina (Osorio, 1969, 1978, 1981, 1990; Osorio \& Ferraro, 1975; Ferraro, 1978; Moberg, 1983; Scutari, 1992,1995; Estrabou et al., 2006; Michlig \& Ferraro, 2012b; Rodriguez et al., 2016). En todo el mundo, se han reportado aproximadamente 70 especies, de distribución principalmente pantropical a subtropical, con algunas especies que se extienden a regiones templadas u oceánicas de las cuales 34 especies han sido halladas en Brasil (Swinscow \& Krog, 1975, 1988; Rogers, 1986; Aptroot, 1987, 2014; Kalb, 1987, 2002; Schumm, 2008; Elix, 2009; Jungbluth, 2010; Mongkolsuk et al., 2012). En Argentina han sido registradas 8 especies del género Pyxine (Calvelo \& Liberatore, 2002; Estrabou et al., 2006; Michlig \& Ferraro, 2012b; Rodriguez et al., 2016); de estas solamente una especie, Pyxine berteriana (Fée) Imshaug, se encuentran registradas en la Provincia de Misiones, con una subespecie Pyxine berteriana (Fée) Imshaug var. subobscurascens (Malme) Imshaug (Calvelo \& Liberatore, 2002).

La Reserva de Biosfera Yaboty (RBY), es un área protegida ubicada en la zona centro-este de la provincia de Misiones (Argentina), en los departamentos Guaraní y San Pedro. Abarca aproximadamente 253.773 ha, en las cuales se encuentran incluidos los Parques Provinciales Caá-Yarí, Esmeralda y Moconá. Esta zona pertenece al Distrito de las Selvas Mixtas de la Provincia Paranaense, Dominio Amazónico (Cabrera, 1994). El clima es cálido y húmedo, con precipitaciones durante todo el año, con un total que varía de $1.564 \mathrm{~mm}$ anuales en Santo
Tomé (Corrientes) a $2.012 \mathrm{~mm}$ en Monteagudo (Misiones). La temperatura media anual varía entre los 20 y 21 grados centígrados, siendo los inviernos suaves y los veranos no excesivamente cálidos debido a las frecuentes lluvias. De mayo a agosto pueden producirse heladas. En el área ubicada al E (mayormente Provincia de Misiones) la vegetación es de tipo selva impenetrable, con dominio en el estrato arbóreo de especies como: laurel (Nectandra saligna (Spreng.) Mez), Guatambú (Balfourodendron riedelianum (Engl.) Engl.), pino paraná (Araucaria angustifolia (Bertol.) Kuntze), yerba mate (Ilex paraguariensis St. Hil.), palo rosa (Aspidosperma polyneuron Mull. Arg.), pindó (Syagrus romanzoffiana (Cham.) Glassman), palmito (Euterpe edulis Mart.), etc. En el estrato arbustivo se encuentran: tacuara brava (Guada trinii (Nees) Nees ex Rupr.), pitanga (Eugenia uniflora L.), ortiga brava (Urera baccifera (L.) Gaudich. Ex wedd.) y numerosas especies de Piper, malváceas, mirtáceas y rubiáceas. En el estrato herbáceo se encuentran gramíneas como Pharus glaber Kunth, Olyra latifolia (L.) Raspail y gran variedad de lianas, orquídeas, helechos, begonias, entre otras. (Cabrera, 1971). Tanto los árboles como los arbustos actúan como primordiales forófitos, en la RBY.

Existen estudios previos que indican que esta reserva constituye un sitio de enorme importancia como área de conservación de muchas especies de líquenes. Si bien en los últimos años el conocimiento de la micobiota de esta Reserva se ha incrementado, muy pocas especies de líquenes se han registrados hasta el momento, las familias estudiadas son: Parmeliaceae (Michlig \& Ferraro, 2012a; Michlig, 2014; Michlig \& Benatti, 2015); Coenogoniaceae (Ferraro \& Michlig, 2013) y Physciaceae (Sarlej et al. 2018).

El propósito de esta publicación es contribuir al conocimiento sobre la diversidad del género Pyxine y de la micobiota liquenizada protegida en la RBY, para posteriores trabajos taxonómicos y ecológicos. Cuatro especies se citan por primera vez para Argentina. Se presentan descripciones de las especies estudiadas, comentarios sobre sus caracteres diagnósticos y diferencias con especies relacionadas, distribución geográfica actualizada e ilustraciones. También se presenta una clave para la identificación de las especies. 


\section{I. Sarlej - Pyxine en la Reserva de la Biosfera Yaboty}

\section{Materiales y Métodos}

Los ejemplares estudiados, se encuentran depositados en el herbario CTES. Se realizó un estudio morfológico de estos ejemplares con microscopio estereoscópico (Leica MZ6). Para determinar el tipo de plecténquima y estudiar la morfología de las ascosporas se realizaron cortes histológicos a mano alzada del talo y de los apotecios respectivamente, los mismos fueron montados en hidróxido de potasio al $5 \%$, para su estudio en microscopio óptico (Leica CME). Las sustancias liquénicas se identificaron mediante reacciones puntuales de color con hidróxido de potasio al $10 \%(\mathrm{~K})$, fluorescencia en cámara de luz UV y cromatografía de capa delgada (CCD) con solvente A, en muestras seleccionadas de cada especie siguiendo el protocolo descripto por Orange et al. (2010).

Los términos utilizados en el trabajo acerca de maculas y pseudocifelas, son considerados siguiendo los criterios de Jungbluth (2010) y para el término dáctilos se siguieron los criterios de Swinscow \& Krog (1988) y Elix (2009), teniendo en cuenta que, en la literatura liquénica, esta última estructura adopta otras denominaciones (Jungbluth, 2010). Abreviaturas utilizadas en material examinado: $\mathrm{PP}=$ Parque Provincial y $\mathrm{RBY}=$ Reserva de Biosfera Yaboty.

\section{Resultados}

La Reserva de la Biosfera Yaboty, es particularmente interesante ya que en ella se encuentra un pequeño porcentaje de la selva que aún existe en la provincia de Misiones. De cinco especies de Pyxine estudiadas, cuatro constituyen primer registro para la Argentina. De esta manera se amplía el número de especies presentes en nuestro país.

\section{Clave para identificación de especies de Pyxine}

1.Talo con dáctilos o soredios.

2. Talo con dáctilos. Pyxine maculata

2'. Talo con soredios.

3. Soralios marginales a submarginales, en ciertos casos desplazándose hacia la lámina; hipotalo negruzco en los ápices de los lóbulos. Pyxine sorediata

3'. Soralios laminares; hipotalo negruzco ausentes.

4. Médula blanca; corteza superior UV + amarillo; máculas ausentes.

Pyxine katendei

4'. Médula amarilla pálida a crema, corteza superior UV-; máculas conspicuas, reticuladas a puntiformes.

Pyxine daedalea

1'. Talo sin dáctilos, ni soredios... Pyxine berteriana

1. Pyxine berteriana (Fée) Imshaug, Trans. Am. Microsc. Soc. 76: 254. 1957. Fig. 1A, B.

$\equiv$ Circinaria berteriana Fée, Ess. Cryptog. Ecorc. Exot. Offic.: 128. 1824.

Talo adnato gris blanquecino, de 2 a $9 \mathrm{~cm}$ de diámetro, lóbulos planos a levemente cóncavos, de 0,4-1 $\mathrm{mm}$ de ancho. Superficie superior con máculas marginales y laminares, conspicuas, irregulares, lineales, reticuladas a puntiformes, con pruina moteada en el ápice de los lóbulos. Médula superior amarilla pálida y médula inferior blanca. Superficie inferior negra en la parte central y más pálidas hacia la periferia, moderadamente ricinada, de distribución agrupada, con una angosta zona marginal sin ricinas; ricinas simples, negras, con ápices blancos. Apotecios moderados en abundancia, de tipo cocoes, planos a levemente cóncavos, de 0,2-1 $\mathrm{mm}$ de diámetro, sésiles, laminares, de margen entero a crenado y sorediado, anfitecio liso, disco color negro epruinoso. Ascosporas de tipo Dirinaria, (13)15-23(25) x (5)7-8 $\mu \mathrm{m}$. Química: Corteza superior K-, UV+ amarillo; médula superior $\mathrm{K}+$ amarilla y médula inferior K-, UV+ amarillo. Metabolitos secundarios: lichenoxantona, atranorina y terpenoides.

Distribución geográfica. Pyxine berteriana es una especie pantropical, ha sido mencionada en Asia, en Tailandia (Mongkolsuk et al., 2012); Australia (Rogers, 1986); África, en Kenia, Tanzania y Uganda (Aptroot, 1987; Swinscow \& Krog, 1988), New Caledonia, Islas Galápagos, América del norte, central y del sur (Aptroot, 1987; Elix, 2009). En Argentina se encuentra en Buenos 
Bol. Soc. Argent. Bot. 54 (2) 2019
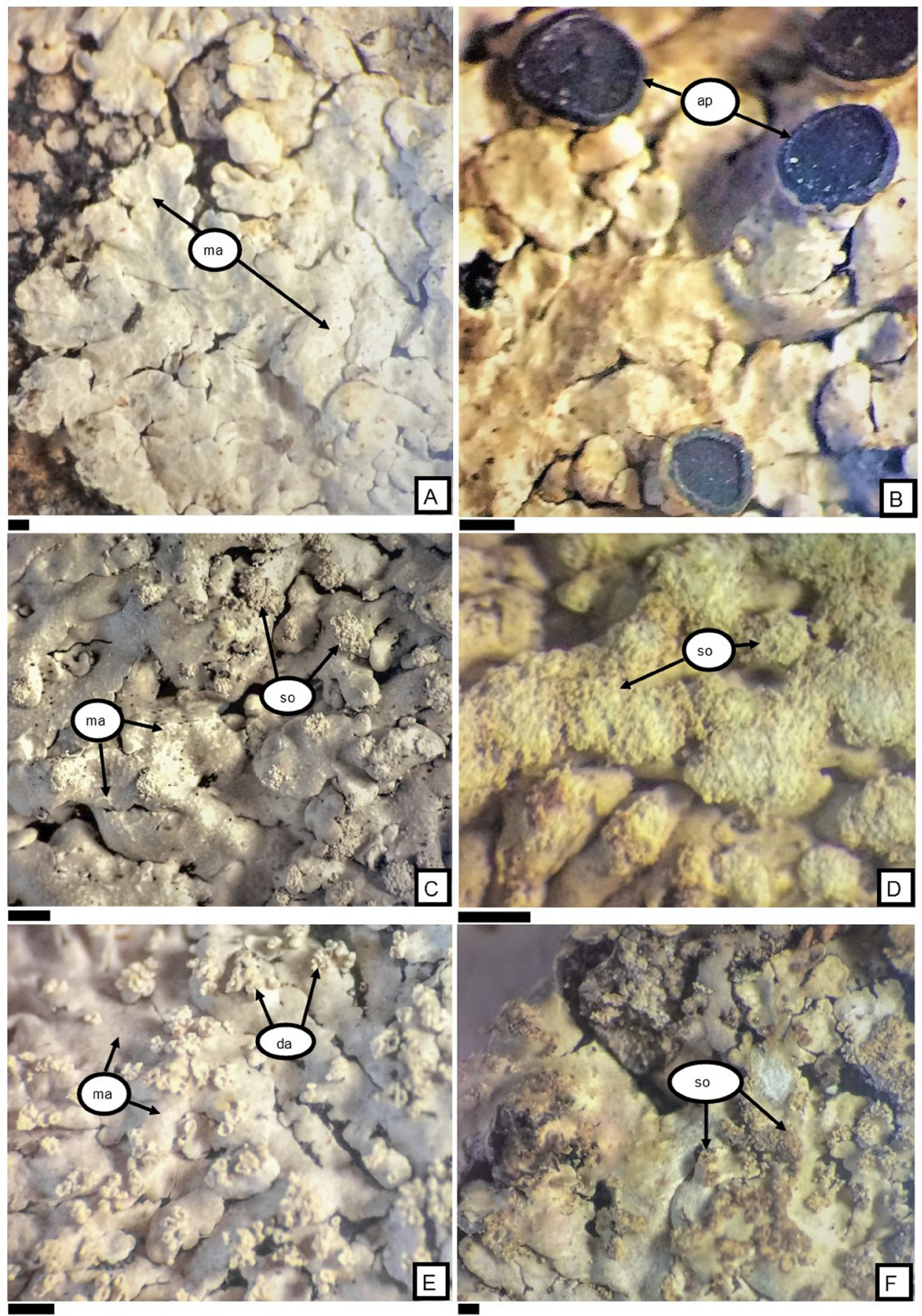

Fig. 1. A: Pyxine berteriana (Michlig et al. 2531), vista de superficie superior del talo. B: Pyxine berteriana (Michlig et al. 2531), detalle de estructura reproductiva. C: Pyxine daedalea (Michlig et al. 1848), vista de superficie superior del talo. D: Pyxine katendei (Michlig et al. 2474), vista de superficie superior del talo. E: Pyxine maculata (Michlig et al. 901), vista de superficie superior del talo. F: Pyxine sorediata (Michlig et al. 931), vista de superficie superior del talo. Abreviaturas= ap: apotecio; da: dáctilos; ma: máculas; so: soralios. Escalas $=\mathrm{A}-\mathrm{F}: 1 \mathrm{~mm}$. 


\section{I. Sarlej - Pyxine en la Reserva de la Biosfera Yaboty}

Aires (Scutari, 1995), Formosa, Jujuy, Misiones (Osorio, 1969, 1978; Calvelo \& Liberatore, 2002), Corrientes (Michlig \& Ferraro, 2012b) y Tucumán (Rodríguez et al., 2016).

Material examinado. ARGENTINA. Prov. Misiones: Dpto. Guaraní, RBY, P.P. Caá-Yarí, metros antes de la casa del guardaparque, sobre rama caída en pastizal, $26^{\circ} 52^{\prime} 19,3$ ' $\mathrm{S}-54^{\circ}$ 13'32,1" W, 531 msnm, 27-III-2010, Michlig et al. 2531 (CTES); Dpto. San Pedro, RBY, PP Moconá, Embarcadero "Piedra Bugre" en tronco, $27^{\circ}$ 9' 13" $\mathrm{S}-53^{\circ} 54$ ' 4", 16-V-2008, Michlig et al. 907 (CTES).

Observaciones. Pyxine berteriana es una especie común en el NEA, se caracteriza por presentar máculas marginales y laminares distribuidas por toda la superficie superior del talo, conspicuas, irregulares, lineales, reticuladas a puntiformes, pruina moteada en el ápice de los lóbulos, la superficie inferior negra en el centro y más pálida hacia la periferia, médula amarilla pálida en la parte superior y en la parte inferior de color blanca, presencia de lichenoxantona en la corteza y en la medula y ausencia de propágulos.

2. Pyxine daedalea Krog \& R. Sant., Thunbergia 2: 7. 1986. Fig. 1C.

Talo gris blanquecino, de 1 a $3 \mathrm{~cm}$ de diámetro. Lóbulos planos a ligeramente convexos, de 0,5-0,7 $\mathrm{mm}$ de ancho, contiguos a imbricados, de ápice redondeado a subtruncado, borde de los lóbulos con hipotalo negruzco. Superficie superior con máculas conspicuas, puntiformes, lineales a reticuladas, epruinosa, con pseudocifelas distribuidas sobre la corteza y soralios de color blanco, laminares, crateriformes a elipsoides, con soredios granulares. Médula amarilla pálida a crema. Superficie inferior negra en la zona central a marrón claro hacia la periferia, abundantemente ricinada, sin zona marginal aparente debido a la distribución homogénea de las ricinas; ricinas negras simples a escuarrosas. Apotecios ausentes. Química: Corteza superior $\mathrm{K}+$ amarillo, UV-; médula $\mathrm{K}+$ amarillo, UV-. Metabolitos secundarios: atranorina y terpenoides.
Distribución geográfica. Pyxine daedalea es una especie pantropical, ha sido mencionada en África, Asia, específicamente en Tailandia (Mongkolsuk et al., 2012), América central, en Costa rica y América del sur, en Brasil (Kalb, 1987; Jungbluth, 2010). Representa el primer registro de la especie para Argentina.

Material examinado. ARGENTINA. Prov. Misiones: Dpto. San Pedro, RBY, PP Moconá, Ruta $\mathrm{N}^{\circ} 2$, sobre rama caída al costado de la ruta, $27^{\circ}$ 9' 13" S - 53 54' 17,2", 315 msnm, 25-V-2009, Michlig \& Niveiro 1848 (CTES).

Observaciones. Pyxine daedalea se caracteriza por presentar en la superficie superior soralios laminares, crateriformes a elipsoides con soredios granulares, máculas conspicuas, puntiformes, lineales a reticuladas, con pseudocifelas sobre la superficie del talo, superficie inferior de color negro en el centro y más pálida hacia la periferia y médula amarilla pálida a crema.

3. Pyxine katendei Swinscow \& Krog, Norw. J. Bot. 22(1): 54. 1975. Fig. $1 D$.

Talo gris mineral, de 1 a $2 \mathrm{~cm}$ de diámetro, muy adherido al substrato. Lóbulos planos algo convexos, subirregulares de 0,5-0,8 $\mathrm{mm}$ de ancho, contiguos, de ápice redondeado a subtruncado. Superficie superior brillosa, emaculada, epruinosa y con pseudocifelas distribuidas sobre el talo, con soralios de color blanco a crema, laminales, crateriformes, orbiculares a elipsoides, soredios farinosos. Médula blanca. Superficie inferior marrón claro y blanca hacia la periferia, moderada a abundantemente ricinada, sin zona marginal aparente, de distribución homogenea; ricinas negras simples. Apotecios ausentes. Química: Corteza superior K-, UV+ amarillo, médula K-, UV-. Metabolitos secundarios: lichenoxanthona $\mathrm{y}$ terpenoides.

Distribución geográfica. Pyxine Katendei es una especie tropical, ha sido mencionada en África en Etiopia, Kenia y Uganda (Swinscow \& Krog, 1975, 1988) y en América del sur en Brasil (Jungbluth, 2010). Constituye el primer registro de la especie para Argentina. 
Material examinado. ARGENTINA. Prov. Misiones: Dpto. Guaraní, RBY, PP Caá-Yarí, metros antes de la casa del guardaparque, $26^{\circ} 52^{\prime} 19,6^{\prime \prime} \mathrm{S}$ $54^{\circ} 13^{\prime} 33,8^{\prime \prime} \mathrm{W}, 526 \mathrm{msnm}, 27-\mathrm{III}-2010$, Michlig et al. 2474 (CTES).

Observaciones. Pyxine katendei se caracteriza por presentar pseudocifelas, soralios laminales de color blanco a crema, crateriformes, orbiculares a elipsoides, la superficie inferior de color marrón claro en el centro y blanca hacia la periferia, la corteza superior UV+ amarillo (lichenoxanthona) y la médula de color blanca.

4. Pyxine maculata Swinscow \& Krog, Norw. J. Bot. 22: 58. 1975. Fig. 1E.

Talo gris verdoso a gris blanquecino, muy brilloso, de 1 a $5 \mathrm{~cm}$ de diámetro. Lóbulos planos a convexos con puntas levemente ascendentes, 0,5-0,7 $\mathrm{mm}$ de ancho, con hipotalo negruzco, contiguos a imbricados, de ápice redondeado a truncado. Superficie superior brillosa, con máculas conspicuas, reticuladas a lineales sobre toda la superficie del talo, con pruina densa hacia la periferia y con pseudocifelas en diferentes partes del talo. Dáctilos marginales a lamínares, no sorediados. Médula blanca a amarilla. Superficie inferior negra a gris oscuro, abundantemente ricinada, sin zona marginal, debido a la distribución homogénea de las ricinas; ricinas negras simples a escuarrosas. Apotecios ausentes. Química: Corteza superior K+ amarillo, UV-; médula K-, UV-. Metabolitos secundarios: atranorina y terpenoides.

Distribución geográfica. Pyxine maculata es una especie tropical, mencionada en África específicamente en Kenia, (Swinscow \& Krog, 1975, 1988). Constituyen el primer registro de la especie para América del sur.

Material examinado. ARGENTINA. Prov. Misiones: Dpto. San Pedro, RBY, PP Moconá, $27^{\circ}$ 9' 13" S - 53 ${ }^{\circ}$ 54' 4", 16-V-2008, Michlig et al. 867 (CTES); ib., embarcadero "Piedra Bugre", $27^{\circ}$ 9' 13" $\mathrm{S}-53^{\circ}$ 54' 4”, 16-V-2008, Michlig et al. 899 (CTES); ib., ipse 901 (CTES).

Observaciones. Pyxine maculata se caracteriza por presentar la superficie superior con máculas conspicuas, reticuladas a lineales sobre toda la superficie del talo, pseudocifelas en diferentes partes de la superficie superior, dáctilos marginales a laminares no sorediadas, superficie inferior de color negro a gris oscuro y médula de color blanca a amarilla.

5. Pyxine sorediata (Ach.) Mont.in Sagra, Hist. Phys. Cuba, Bot. Pl. Cell. 2:188. 1842. Fig. 1F.

$\equiv$ Lecidea sorediata Ach., Syn. Meth. Lich.: 54. 1814.

Talo gris verdoso a gris blanquecino, de 2 a $7 \mathrm{~cm}$ de diámetro. Lóbulos planos a ligeramente cóncavos, con puntas levemente ascendentes de 0,4-1 $\mathrm{mm}$ de ancho, contiguos a imbricados, de ápice redondeado a subtruncado, con hipotalo negruzco. Superficie superior con máculas conspicuas, reticuladas a irregulares, con pseudocifelas, epruinosa o con pruina abundante ubicadas sobre la superficie del talo, en forma de manchas; con soralios de color amarillo a gris oscuro, marginales a submarginales, lineares interrumpidos, en ciertos casos se extiende hacia la lámina presentando formas crateriformes a orbiculares, soredios granulares. Médula amarilla. Superficie inferior de color negro, abundantemente ricinada, de distribución agrupada con angosta zona marginal desnuda hacia la periferia; ricinas negras con ápice blanco, simples. Apotecios ausentes. Química: Corteza superior $\mathrm{K}+$ amarillo, UV-; médula K-, UV-. Metabolitos secundarios: terpenoides.

Distribución geográfica. Pyxine sorediata es una especie pantropical y subtropical (Aptroot, 1987), fue mencionada en el norte, centro y sur de América, Europa, Asia, en África en Etiopia, Kenia y Tanzania (Moberg, 1983; Swinscow \& Krog, 1988, Mongkolsuk et al., 2012), Australia y Asia (Elix, 2009). Constituye el primer registro de la especie, para Argentina.

Material examinado. ARGENTINA. Prov. Misiones: Dpto. Guaraní, RBY, PP Caá-Yarí, mucho antes de la casa del guardaparque, sobre Syagrus romanzoffiana (Cham.) Glassman, $26^{\circ}$ 52' 19,6” S - 54 13' 32,1' W, $531 \mathrm{msnm}, 27$ III-2010, Michlig et al. 2549 (CTES); Dpto. San 


\section{I. Sarlej - Pyxine en la Reserva de la Biosfera Yaboty}

Pedro, RBY, PP Moconá, Sendero "La Gruta", sobre rama caída, $27^{\circ} 9$ ' 13 " S - 53 ${ }^{\circ} 54^{\prime} 4^{\prime \prime} \mathrm{W}, 17$ V-2008, Michlig et al. 931 (CTES).

Observaciones. Pyxine sorediata se caracteriza por presentar lóbulos con hipotalo negruzco, una superficie superior con pseudocifelas distribuidas sobre el talo, con pruina abundante o epruinosa, soralios marginales a submarginales de color amarillo a gris oscuro, lineares interrumpidos, en ciertos casos se extienden hacia la lámina de forma crateriforme a orbicular, su médula es amarilla y la superficie inferior de color negra.

\section{Agradecimientos}

Al Ministerio de Ecología, Recursos Naturales Renovables y Turismo (MERNRyT) de la provincia de Misiones por los permisos de colección y a los guardaparques por la asistencia brindada durante los viajes de campaña. Este trabajo es parte de la tesis de grado de la autora, en la Facultad de Ciencias Exactas y Naturales y Agrimensura de la Universidad Nacional del Nordeste. Se agradece a la Directora Dra. Michlig Andrea y a la Codirectora Dra. Ferraro Lidia por su colaboración y ayuda en el trabajo realizado. A la licenciada María Pía Rodríguez, por la ayuda en la búsqueda bibliográfica. Este trabajo fue financiado por el Consejo Nacional de Investigaciones Científicas y Técnicas (CONICET), la Secretaría General de Ciencia y Técnica (SGCyT-UNNE) y MERNRyT (Proyecto Araucaria XXI).

\section{Bibliografía}

APTROOT, A. 1987. Pyxinaceae (Lichens). In: GÖRTSVAN RIJN, A. R. A. (eds.), Flora of the Guianas, pp 1-59. Koeltz Scientific Books, Koenigstein.

APTROOT, A., P. JUNGBLUTH \& M. E. S. CÁCERES. 2014. A world key to the species of Pyxine with lichexanthone, with a new species from Brazil. Lichenologist 46: 1-4. http://doi.org/10.1017/S002428291400231

CABRERA, A. L. 1971. Fitogeografía de la República Argentina. Bol. Soc. Argent. Bot. 14: 1-42.

CABRERA, A. L. 1994. Regiones fitogeográficas argentinas. In: Kugler, W. F. (ed.), Enciclopedia Argentina de Agricultura y jardinería, pp. 1-85. Acme, Buenos Aires.
CALVELO, S. \& S. LIBERATORE. 2002. Catálogo de los líquenes de la Argentina. Kurtziana 29: 7-170.

ELIX, J. A. 2009: Physciaceae. Pyxine. In: MCCARTHY, P. M. (ed.), Flora of Australia. Lichens, pp. 517-533. ABRS and CSIRO Publishing, Canberra \& Melbourne.

ESTRABOU, C., J. M. RODRIGUEZ, B. PRIERI \& R. LIJTEROFF. 2006. Contribución al conocimiento de los Macroliquenes del extremo sur del Gran Chaco (Argentina). Kurtziana 32: 25-43.

FERRARO, L. I. 1978. Contribución a la Flora liquenológica de Corrientes (República Argentina). Rev. Fac. Cs. Ex. Nat. y Agrim. 2: 167-244.

FERRARO, L \& A. MICHLIG. 2013. New species and additional récords of Coenogonium (Ostropales Coenogoniaceae) from southern South America. Lichenologist 45: 497-504. https://doi.org/10.1017/S0024282913000169

JUNGBLUTH, P. 2010. Estudos taxonómicos em Physcia (Schreb.) Michaux e Pyxine Fries (Physciaceae, Ascomycota) São Paulo. Tesis doctoral. Doctorado em Biodiversidad y médio ambiente. Instituto de Botânica de Secretaria do Estado de Meio Ambiente.

KALB, K. 1987. Brasilianische Flechten, 1. Die Gattung Pyxine. Biblioth. Lichenol. 24: 1-89.

KALB, K. 2002. Pyxine. In: T. NASH III, B. D. RYAN, C. GRIES \& F. BUNGARTZ (eds.), Lichen Flora of the Greater Sonoran. Desert Region, pp. 437-441. Lichens Unlimited, Arizona State University, Tempe.

MICHLIG, A. \& L. I. FERRARO. 2012a. Nuevos registros de especies sorediadas de Parmotrema con ácido protocetrárico (Parmeliaceae Ascomycota) en el sur de Sudamerica. Darwiniana 50: 323-331.

https://doi.org/ 10.2307/41825715

MICHLIG, A. \& L. I. FERRARO. 2012b. Diversidad de macrolíquenes del Parque Nacional Mburucuyá (Corrientes, Argentina). Bol. Soc. Argent. Bot. 47: 287-302.

MICHLIG, A. 2014. Canoparmelia y Crespoa (Parmeliaceae, Ascomycota) en el Nordeste de Argentina; Canoparmelia caroliniana y $C$. cryptochlorophaea nuevas citas para Argentina. Bol. Soc. Argent. Bot. 49: 161-172.

MICHLIG, A. \& M. N. BENATTI. 2015. Revisión of the genus Bulbothrix (Parmeliaceae, lichenized Ascomycota) in NE Argentina, with a key to the species. Cryptog. Mycol. 36: 177-192.

http://doi.org/ 10.7872/crym/v36.iss2.2015.1

MOBERG, R. 1983. Studies on Physciaceae (Lichens) II. The genus Pyxine in Europa. Lichenologist 15: 161-167. http://doi.org/10.1017/S0024282983000250

MONGKOLSUK, P., S. MEESIM, V. POENGSUNGNOEN \& K. KALB. 2012. The lichen family Physciaceae in Thailand I. The genus Pyxine. Phytotaxa 59: 32-54. http://doi.org/10.11646/phytotaxa.59.1.2 
ORANGE, A., P. W. JAMES \& F. J. WHITE. 2010. Microchemical methods for the identification of lichens. 2nd ed. British Lichen Society, London.

OSORIO H. S. \& L. FERRARO. 1975. Contribution to the lichen flora of Argentina VII, and noteworthy records from the province of Corrientes. Comun. Bot. Mus. Hist. Nat. Montevideo 4: 1-4.

OSORIO, H. S. 1969. Contributions to the lichen flora of Argentina, II. Lichens from the province of Misiones. Comun. Bot. Mus. Hist. Nat. Montevideo 4: $1-5$.

OSORIO, H. S. 1978. Contribution to the Lichen Flora of Brazil IV. Lichens from Southern Rio Grande do Sul. Bryologist 81: 452-454.

OSORIO, H. S. 1981. Contribution to the lichen flora of Argentina XIII. Lichens from Misiones province. Comun. Bot. Mus. Hist. Nat. Montevideo 4: 1-18.

OSORIO, H. S. 1990. Contribution to the lichen flora of Argentina XVIII. Lichens from Northwest Argentina. Comun. Bot. Mus. Hist. Nat. Montevideo 5: $1-5$.

ROGERS, R. W. 1986. The genus Pyxine (Physciaceae, lichenized ascomycetes) in Australia. Austral. J. Bot. 34: 131-154. http://doi.org/10.1071/BT9860131

RODRIGUEZ, J. M., J. M. HERNANDEZ, E. FILIPPINI, M. CAÑAS \& C. ESTRABOU. 2016.
Nuevas citas de macroliquenes para Argentina y ampliaciones de distribución en el centro del país. Bol. Soc. Argent. Bot. 51: 405-417.

SARLEJ, M. I., A. MICHLIG \& L. I. FERRARO. 2018. El género Heterodermia (Physciaceae, Lecanoreales) en la Reserva de la Biosfera Yaboty (Misiones, Argentina). Bol. Soc. Argent. Bot. 53: 9-16.

doi: http://doi.org/10.31055/1851.2372.v53. n1.19858

SCUTARI, N. C. 1992. Estudios sobre Pyxinaceae Foliosas (Lecanorales, Ascomycotina) de la Argentina, IV: Clave de los géneros y las especies de la Provincia de Buenos Aires. Bol. Soc. Argent. Bot. 28: 169-173.

SCUTARI, N. C. 1995. Los macrolíquenes de Buenos Aires, II: Phaeophyscia, Physcia y Pyxine (Physciaceae, Ascomycotina). Darwiniana 33: 211-231.

SCHUMM, F. 2008. Flechten Madeiras, der Kanarenund Azoren. Wangen.

SWINSCOW, T. D. V. \& H. KROG. 1975. The genus Pyxine in East Africa. Norw. J. Bot. 22: 43-68.

SWINSCOW, T. D. V. \& H. KROG. 1988. Macrolichens of East Africa. British Museum, London. https://doi.org/10.1017/S0266467400003771 